\title{
Comparison of Acoustic Impedance Eduction Techniques for Locally-Reacting Liners
}

\author{
M. G. Jones,, T. L. Parrott, ${ }^{\ddagger}$ W. R. Watson ${ }^{\S}$ \\ NASA Langley Research Center, Hampton, VA 23681-2199
}

\begin{abstract}
$\underline{\text { Abstract }}$
Typical acoustic liners used in current aircraft inlets and aft-fan ducts consist of some type of perforated facesheet bonded to a honeycomb core. A number of techniques for determining the acoustic impedance of these locallyreacting liners have been developed over the last five decades. In addition, a number of models have been developed to predict the acoustic impedance of locallyreacting liners in the presence of grazing flow, and to use that information together with aeroacoustic propagation codes to assess the noise absorption provided by these liners. These prediction models have incorporated the results from databases acquired with specific impedance eduction techniques. Thus, while these prediction models are acceptable for liners that are similar to those tested in these databases, their application to new liner configurations must be viewed with caution.

The primary purpose of this paper is to provide a comparison of impedance eduction techniques that have been implemented at various aerospace research laboratories in the United States (NASA Langley Research Center, General Electric Aircraft Engines, B. F. Goodrich and Boeing). A secondary purpose is to provide data for liner configurations that extend the porosity range beyond that which has been previously used in common aircraft engine nacelles. Two sets of liners were designed to study the effects of three parameters: perforate hole diameter, facesheet thickness and porosity. These two sets of liners were constructed for testing in each of the laboratories listed above. The first set of liners was designed to fit into the NASA Langley and Boeing test facilities. The second set was designed to fit into the General Electric Aircraft Engines and B. F. Goodrich test facilities. By using the same parent material, both sets of liners were identical to within the limits of material and fabrication variability. Baseline data were obtained in the normal incidence impedance tubes at NASA Langley and B. F. Goodrich. The results were found to compare extremely well. The

\footnotetext{
${ }^{\dagger}$ Research Scientist, Structural Acoustics Branch, Aerodynamics, Aerothermodynamics and Acoustics Competency, Member of AIAA

${ }^{\ddagger}$ Senior Research Scientist, Structural Acoustics Branch, Aerodynamics, Aerothermodynamics and Acoustics Competency

${ }^{\S}$ Senior Research Scientist, Computational Modeling and Simulation Branch, Aerodynamics, Aerothermodynamics and Acoustics Competency, Senior member of AIAA
}

samples were then tested in the grazing flow ducts of each of the four laboratories. Perhaps the most significant result of these comparisons is that the educed acoustic resistances for the liners used in this study increase as the mean flow profile is modified from uniform to 3-D shear. This realization has demonstrated the need for an frequency-dependent impedance eduction technique that incorporates 3-D shear flow and is efficient.

\section{Nomenclature}

$c_{0} \quad$ ambient sound speed, $\mathrm{m} / \mathrm{s}$

$D$ hole diameter, $\mathrm{mm}$

$f$ frequency, $\mathrm{Hz}$

$h \quad$ cavity height, $\mathrm{mm}$

$H$ duct height, m

$i \quad \sqrt{-1}$

$p(x, y)$ complex acoustic pressure, $\mathrm{Pa}$

$p_{\text {ref }} \quad$ reference pressure, $20 \mu \mathrm{Pa}$

$P_{\mathrm{s}} \quad$ static pressure, $\mathrm{kg} /\left(\mathrm{m} \cdot \mathrm{s}^{2}\right)$

$P_{\mathrm{t}} \quad$ total pressure, $\mathrm{kg} /\left(\mathrm{m} \cdot \mathrm{s}^{2}\right)$

POA percent open area, $\%$

$R_{f} \quad$ DC flow resistance, $\mathrm{kg} /\left(\mathrm{m}^{2} \cdot \mathrm{s}\right)$

$t$ time, s

$T$ facesheet thickness, mm

$x_{i} \quad$ wall measurement axial location, $m$

Symbols:

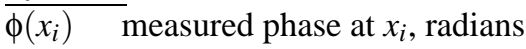

$\rho_{0} \quad$ ambient density

$\theta \quad$ normalized acoustic resistance

$\omega \quad$ angular frequency $(=2 \pi f)$

$\chi \quad$ normalized acoustic reactance

$\zeta \quad \theta+i \chi$, normal incidence acoustic

Abbreviations: impedance, normalized by $\rho_{0} c$

$\begin{array}{ll}\text { FEM } & \text { Finite Element Method } \\ \text { FEMS } & \text { Finite Element Method with Shear } \\ \text { FRM } & \text { Flow Resistance Method } \\ \text { GFAZ } & \text { Grazing Flow Acoustic Impedance } \\ & \text { Method } \\ \text { IL } & \text { Insertion Loss } \\ \text { ILM } & \text { Insertion Loss Method } \\ \text { SMM } & \text { Single Mode Method } \\ \text { SPL }\left(x_{i}\right) & \text { measured sound pressure level at } x_{i}, \mathrm{~dB} \\ e^{i \omega t} \text { time convention is used throughout this paper. }\end{array}$




\section{Introduction}

Continual increases in aircraft traffic place an increasing emphasis on noise abatement research. One critical component of noise abatement methodology is the use of passive acoustic liners that are installed in the walls of the engine nacelle inlets and aft-fan ducts. Numerous investigations have been conducted over the last five decades to develop prediction models for locally-reacting acoustic liners. ${ }^{1-8}$ These models have been based on extensive experimental data acquired with a variety of impedance eduction techniques. Thus, proper evaluation of this data requires a thorough understanding of the underlying assumptions in each impedance eduction technique. Only then can these results, together with appropriate aeroacoustic propagation codes, be confidently used to determine the expected noise reductions of selected liner configurations. In addition, with the recent trend toward shorter aircraft engines with higher bypass ratios, the need for increased accuracy in the design and fabrication of optimized passive liners continues to increase.

In 1997, a group of U.S. aerospace research laboratories decided to conduct a study to compare the results achieved with their respective impedance eduction techniques. Each of the techniques has strengths and weaknesses that are functions of their underlying assumptions and the availability of appropriate facilities and instrumentation. For example, the best technique ${ }^{9}$ used at NASA Langley (a finite element method coupled with an optimizer) to educe the acoustic impedance of liners in the presence of grazing flow is labor and computationintensive. Efforts are underway to signifcantly increase the efficiency of this technique and to assess the quality of impedance eduction techniques in use at other facilities. In addition, there has been a recent interest in the use of perforate liners with geometric parameters outside of the range of the previous exhaustive experimental databases. ${ }^{10}$

The primary purpose of the current report is to provide a comparison of a number of impedance eduction techniques that have been implemented at various aerospace laboratories in the United States (NASA Langley Research Center, General Electric Aircraft Engines, B. F. Goodrich and Boeing). A secondary purpose is to provide data for liner configurations that extend the porosity range beyond that previously used in common aircraft engine nacelles. Two sets of liners were designed to study the effects of the following key parameters: perforate hole diameter, facesheet thickness and porosity. These two sets of liners were constructed for testing in the test facilities of each of the laboratories listed above. The first set of liners was designed to fit into the NASA Langley and Boeing test facilities, while the second set was designed to fit into the General Elec-

\begin{tabular}{|c||c|c|c|c|}
\hline Configuration & 1 & 2 & 3 & 4 \\
\hline POA (\%) & 8.7 & 6.4 & 13.2 & 13.0 \\
\hline Hole Diameter, D & 0.99 & 0.99 & 0.99 & 2.36 \\
\hline Sheet Thickness, T & 0.64 & 0.64 & 0.64 & 0.81 \\
\hline
\end{tabular}

Table 1. Acoustic Liner Configurations (distance units in $\mathrm{mm}$, cavity height of $38.1 \mathrm{~mm}$ for each liner)

tric and B. F. Goodrich test facilities. By using the same parent material, the acoustic properties of both sets of liners were made to be as identical as possible.

In this report, results are provided for four liner configurations that consist of punched aluminum facesheets bonded to hexcell honeycomb cavities. These test panels were selected to demonstrate the effects of perforate facesheet hole diameter, thickness and porosity (commonly provided in terms of percent open area). For each configuration, two test panels were fabricated for each pair of test facilities. The first was designed to be tested in a normal incidence tube, which is used to determine the normal incidence acoustic impedance of the test panel. A second panel, fabricated using the same material, was designed such that it could be mounted into a grazing incidence impedance tube, which is used to educe the normal incidence acoustic impedance of the test panel in the presence of grazing flow. As a baseline comparison, the samples were tested in the normal incidence impedance tubes at NASA Langley and B. F. Goodrich. The samples were then tested in the grazing flow ducts of each of the four laboratories.

The remainder of this paper is organized into four sections. The first section gives a description of the liner configurations used in this investigation. The second section describes the test facilities at each of the participating laboratories, and the impedance eduction techniques used at each of these facilities. The third section contains a comparison of results obtained in each facility, together with a discussion of these results. Conclusions relevant to this investigation are presented in the final section.

\section{Description of Test Liners}

Four liner configurations were tested in this investigation. Each of these consisted of a perforated facesheet (punched aluminum) bonded onto $9.5 \mathrm{~mm}$-diameter hexcell honeycomb cavities. Table 1 contains a list of geometric parameters for each of the four configurations. Four test panels were fabricated from the same parent material for each of the configurations (total of 16 panels). The first had dimensions of $50.8 \mathrm{~mm} \times 50.8 \mathrm{~mm}$, and was designed to be mounted onto the NASA Langley normal incidence tube. The second had dimensions of $127.0 \mathrm{~mm} \times 127.0 \mathrm{~mm}$, and was designed to be 


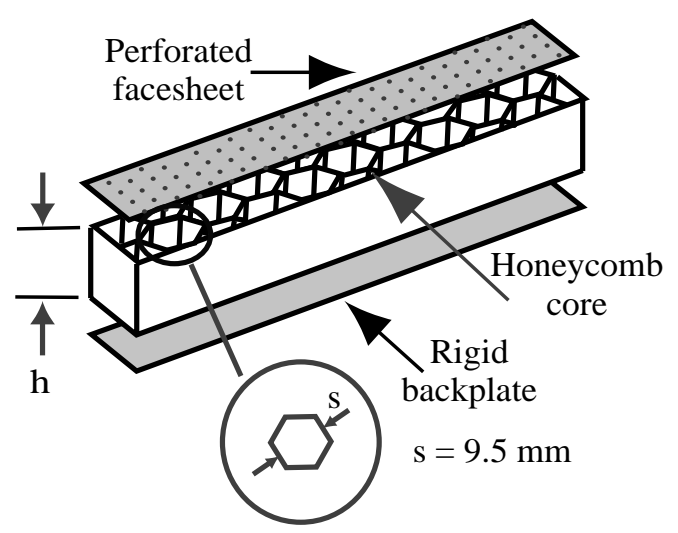

Figure 1. Sketch of test liner.

mounted onto the B. F. Goodrich normal incidence tube. These normal incidence tubes were used to determine the normal incidence acoustic impedance of the test panels. A third panel, with dimensions of $50.8 \mathrm{~mm} \times 406 \mathrm{~mm}$, was designed to mount in the NASA Langley and Boeing flow ducts. The last panel had dimensions of $139.7 \mathrm{~mm} \times 609.6 \mathrm{~mm}$, and was designed to mount in the General Electric and B. F. Goodrich flow ducts. These ducts are used to educe the impedance of the test panel in the presence of grazing flow.

Figure 1 contains a sketch of the $50.8 \mathrm{~mm} \times 406 \mathrm{~mm}$ panel. As indicated in table 1, the percent open area (POA), hole diameter (D) and facesheet thickness (T) ranges are

$$
\begin{gathered}
6.4 \%<P O A<13.2 \% \\
0.99 \mathrm{~mm}<D<2.36 \mathrm{~mm} \\
0.64 \mathrm{~mm}<T<0.81 \mathrm{~mm}
\end{gathered}
$$

The hole diameters and facesheet thicknesses are within the range of those commonly in use in current aircraft. The percent open area range, on the other hand, extends beyond that which has been typically used in aircraft.

\section{Test Facilities}

\section{NASA Langley Research Center}

The NASA Langley Research Center grazing incidence tube was used in the current investigation to educe the normal incidence acoustic impedance of the test panel in the presence of grazing incidence sound and mean flow. A schematic of this waveguide is provided in figure 2 . This apparatus has a $50.8 \mathrm{~mm} \times 50.8 \mathrm{~mm}$ crosssection in which a controlled aeroacoustic environment is achieved. The $50.8 \mathrm{~mm}$-wide $\times 406 \mathrm{~mm}$-long liner is centered in a test section that includes the region from the source plane (203 $\mathrm{mm}$ upstream of the liner leading edge) to the exit plane ( $203 \mathrm{~mm}$ downstream of the liner trailing edge).

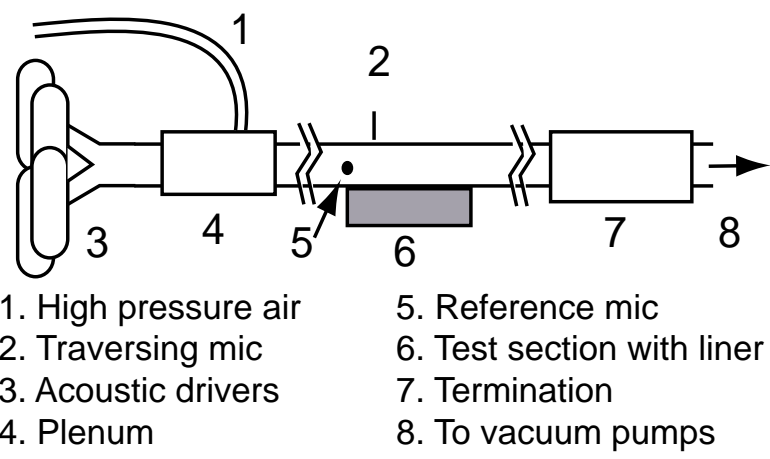

Figure 2. NASA Grazing Incidence Tube.

For the current investigation, tests were conducted with discrete tones (one at a time) from 1.0 to $3.0 \mathrm{kHz}$, with sound pressure levels of $130 \mathrm{~dB}$ at the liner leading edge. The uniform flow Mach number used to perform each impedance eduction in this report was taken to be the average value of the Mach number profile measured at the mid-liner axial plane $(406 \mathrm{~mm}$ downstream of the source plane). Tests were conducted for centerline Mach numbers of 0.0, 0.1, 0.3 and 0.5. Acoustic waves are propagated from left to right in figure 2 , across the surface of the test panel, and into a termination section designed to minimize reflections over the frequency range of interest.

Two $6.35 \mathrm{~mm}$-diameter condenser-type microphones are used to acquire the complex acoustic pressure data. The first is a reference microphone, which is flushmounted on the side wall at the test panel leading edge. The amplitude of the discrete tone supplied by the acoustic drivers was controlled to achieve the desired sound pressure level at this location. The second microphone is flush-mounted on an axial traverse bar, which forms a portion of the upper wall of the test section. A $13 \mathrm{~mm}$ wide precision-machined slot in the top wall of the flow impedance tube allows this axial traverse bar to traverse the test section length by means of a computer-controlled digital stepping motor. The data acquisition program automatically positions the traversing microphone at preselected locations, $x_{i}$, from $203 \mathrm{~mm}$ upstream of the leading edge to $203 \mathrm{~mm}$ downstream of the trailing edge of the liner. At each measurement location, a transfer function between the traversing and reference microphones is used to determine the sound pressure level $\operatorname{SPL}\left(x_{i}\right)$ and phase $\phi\left(x_{i}\right)$ relative to the fixed reference microphone location. The complex acoustic pressure at a given axial wall location is determined from the equation

$$
p\left(x_{i}, H\right)=p_{\text {ref }} 10^{S P L\left(x_{i}\right) / 20} e^{i \phi\left(x_{i}\right)}
$$

where the reference pressure, $p_{\text {ref }}$, is $20 \mu \mathrm{Pa}$. 
Three techniques were used to determine acoustic impedance of the test liner. These methods have been discussed at length in previous papers; ${ }^{11,12}$ thus, only enough detail is presented in this paper to provide a comparison with the techniques used by the other investigating partners. The single mode method (SMM) educes the impedance from the uniform flow impedance boundary condition ${ }^{13}$ along the sample surface, using the mode shape for a single progressive wave. The measured data are used to determine the axial and transverse wave numbers of the purely progressive mode. This method assumes the mean flow to be uniform.

The finite element method (FEM) also uses the uniform flow assumption. This method iterates on the numerical solution to the 2-D convected wave equation to determine an impedance that reproduces the measured amplitudes and phases of the upper wall complex acoustic pressures. The FEM uses the measured exit impedance and an assumed plane wave source as boundary conditions. Cubic polynomial basis functions and the uniform flow impedance boundary condition ${ }^{13}$ are used in this method.

The finite element method with shear (FEMS) uses a similar procedure to the FEM, but it solves the 2-D linearized equations of mass and momentum continuity using a linear element. A parallel shear flow (sometimes referred to as 1-D shear flow in that it varies in transverse direction, but not in axial direction) is incorporated into this method. The source and exit planes for the FEMS are assumed to be within the lined section of duct and the input data for these planes are obtained using a single progressive mode assumption. (Ideally, these boundary conditions would be measured with transverse probes, but these intrusive measurements were determined to be unacceptable at this time.) However, a no-slip sheared flow impedance boundary condition is applied for this model. It should be noted that in a Mach 0.0 environment, the SMM and FEMS have identical data inputs. However, the SMM solves a Helmholtz's equation and the FEMS solves the linearized equations of mass and momentum (for zero flow). Differences between the finite element discretization used in the FEMS and the modal description used in the SMM may cause slightly different results for the zero flow condition. Similarly, differences between the FEM and the FEMS in zero flow may be attributed to discretization error in the governing equations due to the use of cubic and linear finite elements, respectively.

\section{Boeing}

The Boeing Wichita Noise Lab Grazing Flow Facility also has a $50.8 \mathrm{~mm} \times 50.8 \mathrm{~mm}$ cross-section, and can be used to test liners with dimensions of $50.8 \mathrm{~mm} \times 406 \mathrm{~mm}$. The surface of the test liner forms the upper wall of the duct, and the traversing microphone is flush-mounted in a teflon strip that forms a portion of the lower wall of the duct. For the data shown in this report, this microphone was traversed over the length of the test liner. Aluminum tape was used to "hardwall" (i.e., to cover a portion of the liner such that it appeared acoustically "hard") regions near the leading and trailing edges of the liner, such that these measurements could be made in hardwall sections upstream and downstream of the softwall section. A reference microphone is located in the lower wall, in the plane of the leading edge of the lined section, and is offset $12.7 \mathrm{~mm}$ from the traversing microphone.

An acoustic signal is generated by an electropneumatic acoustic driver and is coupled to the duct via an exponential acoustic horn. As the traversing microphone is positioned over the length of the liner, a transfer function between the two microphones is used to determine the complex acoustic pressure profile in this section. These measurements are acquired at a total of 80 microphone locations spaced $5.1 \mathrm{~mm}$ apart. In the hardwall sections upstream and downstream of the liner, only plane waves will propagate for frequencies up to approximately $3 \mathrm{kHz}$ (depending on grazing flow Mach number). For the current study, the acoustic driver was used to generate multitone signals. These signals contained 160 tones from 1008 to $6096 \mathrm{~Hz}$, in $32 \mathrm{~Hz}$ increments. For the purposes of the current investigation, only those results for frequencies up to approximately $3 \mathrm{kHz}$ are reported in order that the results can be compared with those from the other facilities on a similar basis.

A grazing flow data analysis program (GFAZ) is used to determine the acoustic impedance based on the measured complex acoustic pressure profile. The analysis program assumes multiple (up to 8) modes are present in the regions upstream and downstream of the liner, as well as in the lined section. The GFAZ program conducts separate computations in each of these three regions, then matches the acoustic pressure and particle velocity across the interfaces to determine the modal amplitudes in each of the regions. This analysis also assumes the mean flow is uniform (assumed equal to the centerline Mach number for this study) and the termination is nonreflecting. The tunnel diffuser is filled with bulk acoustic absorber material to minimize reflections such that the latter assumption is reasonably correct. Based on an assumed value of the liner impedance, a complex acoustic pressure profile is computed. This predicted acoustic pressure profile is compared with the measured acoustic pressure profile, and the predicted impedance is assumed to be correct when the two acoustic pressure profiles are matched to within an acceptable tolerance.

It is important to also note that this multi-tone source will cause the rms acoustic particle velocity to be higher than that present for a single tone source, if the sound 
Boundary Layer $\mathrm{P}_{\mathrm{t}}$ Probe

$\& \mathrm{P}_{\mathrm{S}}$ at the DC Flow Sample

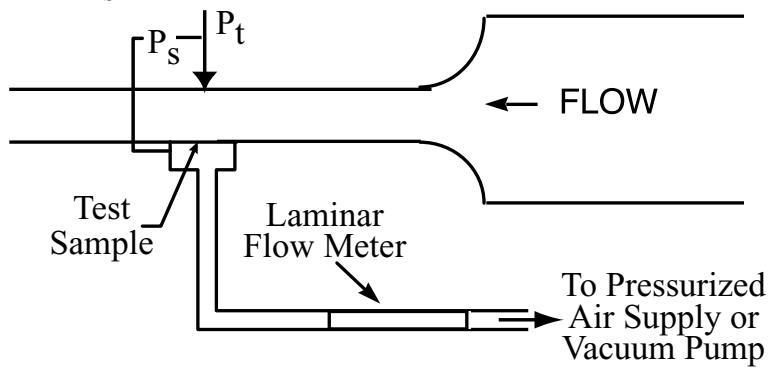

Figure 3. General Electric Flow Duct.

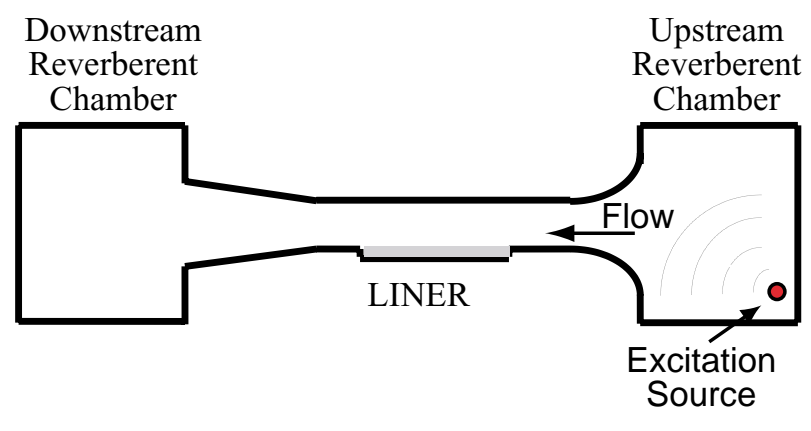

Figure 4. B. F. Goodrich Flow Duct. pressure levels of the individual tones are approximately equal to that of the single tone. For linear liners, this affect the educed impedance. However, for nonlinear liners (i.e.; where impedance is a function of particle velocity), results acquired with this technique should not be expected to match those acquired with single-tone methodologies. Thorough descriptions of the analysis methodology and the Boeing flow duct facility are provided in the references. ${ }^{10,14,15}$ This method is referenced as the GFAZ method in the remainder of this paper.

\section{General Electric}

The flow duct apparatus in the Acoustics Laboratory at General Electric was designed to measure flow resistance $\left(R_{f}\right)$ in the presence of grazing flow. This device (figure 3) allows bias flow air to be "pushed" or "pulled" through the facesheet material that is mounted flush in the flow duct wall. The flow resistance, which is the ratio of the pressure drop across the sample to the velocity of the bias flow through the sample, was measured for incident bias flow velocities of 250 to $-150 \mathrm{~cm} / \mathrm{sec}$ (positive for "push", negative for "pull"), with grazing flow Mach numbers up to 0.7 .

For "thin" resistive specimens, it is assumed that the flow resistance measured at a particular bias flow velocity is equivalent to the acoustic resistance ('rms'-derived) of the sample that would be present if the sample were exposed to an acoustic particle velocity of the same magnitude. In other words, a sinusoidal acoustic particle velocity distribution (as a function of time) that impinges on the surface of a liner can be discretized into " $n$ " acoustic particle velocities, and the corresponding " $\mathrm{n}$ " bias flow velocities can be used to acquire a series of flow resistances. An estimate of the rms acoustic resistance due to the sinusoidal acoustic particle velocity signal is achieved by computing the rms flow resistance from these bias flow velocities.

Clearly, since the honeycomb and back-plate are removed from the sample for this test, only the acoustic resistance properties can be deduced from this method. Also, this methodology does not provide any frequencydependent characteristics of the acoustic resistance. However, this method is quite simple and quick. It also implicitly accounts for 3-D boundary layer effects. A more detailed discussion of this flow resistance method (FRM) is provided by Syed. ${ }^{16}$

\section{B. F. Goodrich}

The B. F. Goodrich methodology relies on insertion loss (IL) measurements to educe the impedance of a liner. These measurements were conducted in a flow duct apparatus (figure 4) with a $139.7 \mathrm{~mm} \times 101.6 \mathrm{~mm}$ crosssection. This apparatus consists of two reverberation chambers connected by a rectangular flow duct. For this methodology, the IL is defined as the difference between the sound pressure levels in the diffuse regions of the upstream and downstream reverberation chambers. The acoustic power suppression of a particular test liner installed in the test section is taken to be equal to the difference between the IL for the lined condition and the IL for a hardwall condition (i.e., liner replaced with a hard wall).

A 2-D modal propagation method that assumes a uniform mean flow is used to determine the frequencydependent acoustic impedance of the test liner. This method requires knowledge of the modal coefficients in the hardwall section upstream of the lined region. For this purpose, "calibration" liners are tested first in the IL apparatus. The impedance is known for these liners; thus, the modes that are present in the upstream section can be deduced using the modal propagation method. If the unknown test materials are similar to the "calibration" liners (true for liners used in current study), the upstream incident modal pattern is assumed to remain the same for the test liner as it was for the "calibration" liner. With the modal pattern of the source properly defined, the modal propagation method can be used to iterate the impedance boundary condition (due to the test liner). For this methodology, an impedance prediction 
model that includes displacement thickness as a parameter (i.e., parallel, or 1-D, shear flow assumption) is used to select the impedances that are to be included in this iterative scheme. The impedance at which the predicted IL most closely matches the measured insertion loss is taken to be an estimate of the correct liner impedance. A more detailed discussion of this Insertion Loss Method (ILM) is provided by Syed. ${ }^{16}$

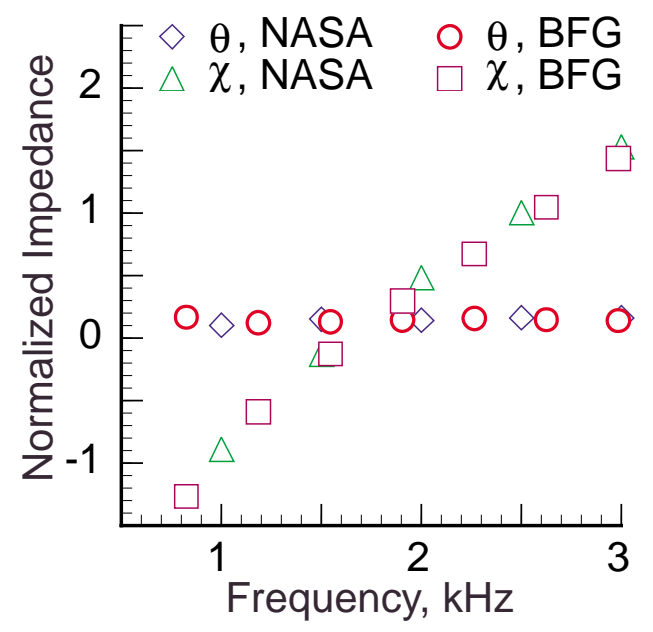

Figure 5. Normal Incidence Impedance, Liner 1 - NASA Langley \& B. F. Goodrich.

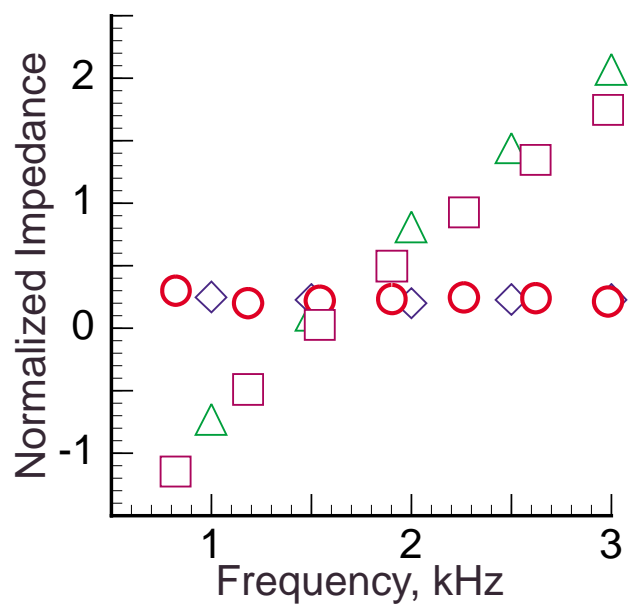

Figure 6. Normal Incidence Impedance, Liner 2 - NASA Langley \& B. F. Goodrich.

\section{Results and Discussion}

Prior to implementing the various grazing flow impedance eduction techniques described in the previous section, the smaller samples were tested over similar

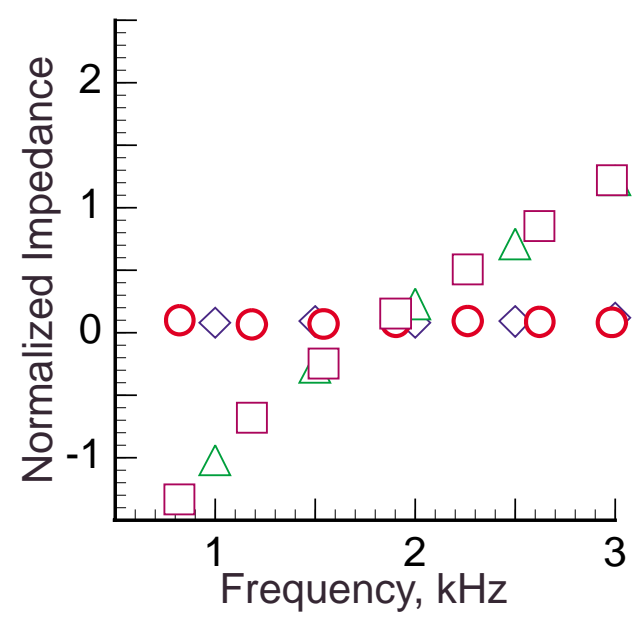

Figure 7. Normal Incidence Impedance, Liner 3 - NASA Langley \& B. F. Goodrich.

frequency ranges $(1.0 \leq f \leq 3.0 \mathrm{kHz}$, in steps of $0.5 \mathrm{kHz}$ for NASA Langley; $0.824 \leq f \leq 2.984 \mathrm{kHz}$, in steps of $0.360 \mathrm{kHz}$ for B. F. Goodrich) in the normal incidence impedance tubes at NASA Langley Research Center and B. F. Goodrich. As shown in figures 5, 6, 7 and 8, the results were very well matched. It was expected that the grazing flow normal incidence impedances for zero grazing flow would be nearly identical to the normal incidence tube values. For those laboratories where the Mach 0.0 condition was tested in a grazing flow duct, this assumption was demonstrated to be valid. ${ }^{12}$

Figures 9, 10 and 11 provide comparisons of the normalized acoustic impedances educed with each of the six methods for liners 1 and 3 at a mean flow Mach number of 0.3. (Note: Due to cost and time constraints, some liners could not be tested with the ILM and GFAZ.) A number of observations can be drawn from the resistances in figure 9 for liner 1. The ILM results are observed to be slightly higher than the SMM and FEM results, which are reasonably well matched. The FEMS results are noticeably higher than those of the ILM, and the FRM results are even higher.

In general, the GFAZ results are higher than any of the others. Recall that this method is implemented with a multi-tone source, whereas the others are implemented with single-tone sources. As a result, the rms acoustic particle velocity for the GFAZ data is higher than that for the other methods. Also, the uniform flow Mach number used in the GFAZ method was taken to be equal to the centerline Mach number, whereas the average Mach number was used for the other uniform flow methods. Thus, the increase in acoustic resistance observed with the GFAZ method is likely due to increases in acoustic particle velocity and mean flow velocity bias, as might 


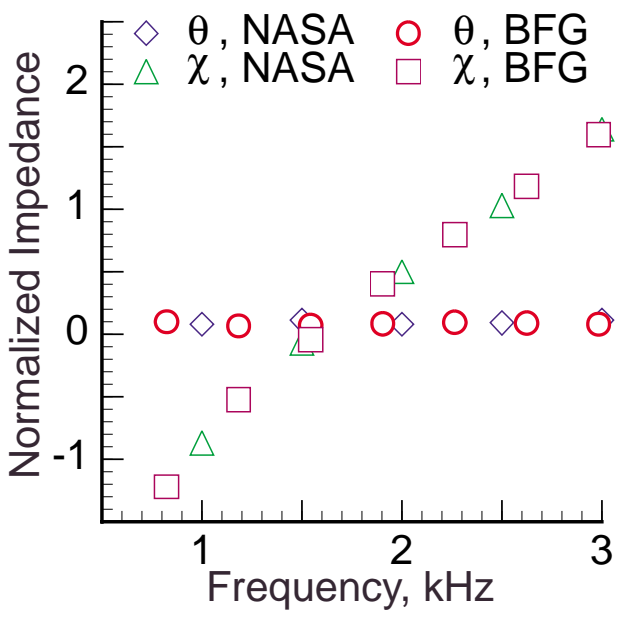

Figure 8. Normal Incidence Impedance, Liner 4 - NASA Langley \& B. F. Goodrich.

be expected. In summary, the key observations can be explained as follows:

1. The SMM and FEM methods both assume the mean flow to be uniform. While the SMM method can only be implemented when the sound field over the lined region is dominated by a single mode, this method should be expected to give similar results to the FEM method when this condition is satisfied.

2. The ILM uses a 2-D modal propagation technique with a uniform mean flow to educe the impedance of a test liner. However, initial estimates of this impedance are obtained using a prediction model that assumes a 1-D (parallel) shear flow. Consequently, it has been suggested ${ }^{16}$ that, in some sense, this methodology incorporates 1-D shearflow physics.

3. The FEMS method assumes 1-D (parallel) shear flow, with inflow and outflow boundary conditions approximated from a single progressive mode assumption.

4. The Mach number is used as a test parameter in the FRM, but is not explicitly included in the calculations. Therefore, since the flow resistance is measured in a grazing flow duct that clearly contains 3-D shear flow, the FRM implicitly assumes a 3-D shear flow profile.

The results described for liner 1 generally hold for liner 3, with one exception. For this liner, the GFAZ method gives results that are very similar to those acquired with the other uniform flow techniques (SMM,
FEM and ILM), except at the highest frequency. These findings suggest that the significance of the differences between the mean flow profiles used in the various techniques decreases as the percent open area (POA) is increased from 8.7 to $13.2 \%$.

These observations suggest the resistance increases toward the FRM values as more details of the mean flow profile are included. However, the FRM does not provide the frequency-dependent characteristics of the acoustic impedance. It also does not compute reactance. In an attempt to overcome these limitations, the FEMS is currently being enhanced to incorporate 3-D shear flow.

Figure 10 contains the reactances educed with each of the techniques (the FRM does not provide reactances). In general, the values for the techniques implemented with single-tone sources are observed to be well matched over the frequency range of interest, with one exception. The reactance values educed with the FEMS method are lower than those of the other methods at the lower end of the frequency spectrum. Similar results observed for liner 3 are not included for the sake of brevity.

In general, results for the Mach 0.5 condition (figures 12,13 and 14) are similar to those described above for the Mach 0.3 condition. The three methods that incorporate uniform flow (SMM, FEM and ILM) provide nearly identical results. For this flow velocity, however, the FEMS acoustic resistance values are observed to be higher than those of the FRM. The acoustic reactance results, however, are observed to vary more dramatically between the impedance eduction techniques for this flow condition. As shown in figure 13, the acoustic reactances educed with the FEM are higher than those of the SMM and ILM, which are in turn higher than those of the FEMS. It is unfortunate that only the FEMS is fully implemented using the parallel shear flow assumption and, therefore, cannot be fully validated without the development of an additional methodology. As stated earlier, a 3-D shear flow version of the FEM is currently being developed in an attempt to fill this void. For completeness, tables 2, 3, 4 and 5 contain acoustic impedances educed with each of the techniques for which data were acquired.

\section{Conclusions}

1. The normal incidence acoustic impedances measured in the normal incidence tubes of NASA Langley and B. F. Goodrich are well matched.

2. In general, agreement between the various impedance eduction techniques is driven by the choice of mean flow profile. For example, techniques that assume uniform flow tend to agree.

3. As more details of the mean flow profile are incorporated (uniform to parallel shear to 3-D shear), the educed resistance increases. 


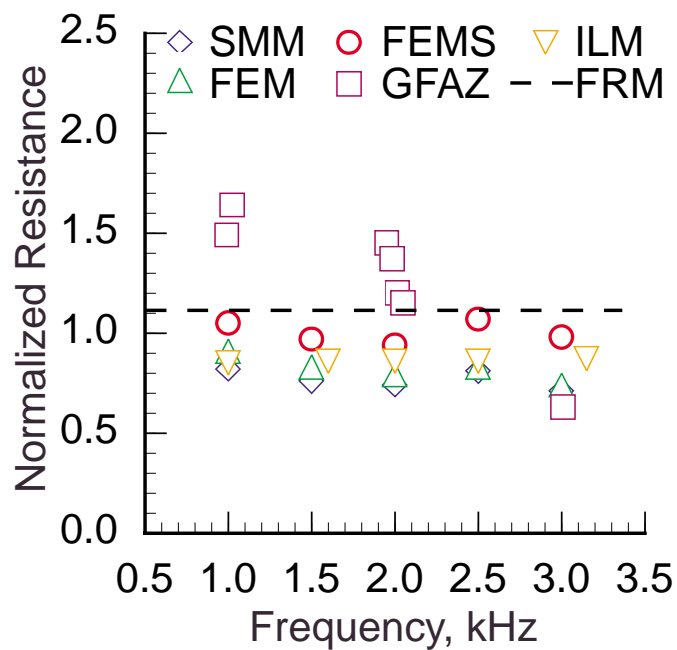

Figure 9. Liner 1 Resistance, $M=0.3$.

4. The development of a 3-D shear flow impedance eduction technique that provides frequencydependent impedance eductions is needed. NASA Langley is currently developing this technique.

\section{References}

1. Ingard, U., "On The Theory And Design Of Acoustic Resonators," Journal of Acoustical Society of America, Vol. 25, No. 6, 1953.

2. Rice, E.J., "A Model For The Acoustic Impedance Of A Perforated Plate Liner With Multiple Frequency Excitation.” NASA TMX-67950, 1971.

3. Melling, T.H., "The Acoustic Impedance of Perforates at Medium and High Sound Pressure Levels." Journal of Sound and Vibration, Vol. 29, No. 1, 1973.

4. Guess, A.W., "Calculation Of Perforated Plate Liner Parameters From Specified Acoustic Resistance And Reactance," Journal of Sound and Vibration, Vol. 40, No. 1, 1975.

5. Hersh, A.S., Walker, B., and Bucka, M., "Effect Of Grazing Flow On The Acoustic Impedance Of Helmholtz Resonators Consisting Of Single And Clustered Orifices." AIAA 78-1124, 1978.

6. "Aeroacoustics of Flight Vehicles: Theory and Practice - Volume 1: Noise Sources," NASA RP-1258, edited by H.H. Hubbard, 1991.

7. Parrott, T. L. and Jones, M. G., "Parallel-element Liner Impedances for Improved Absorption of

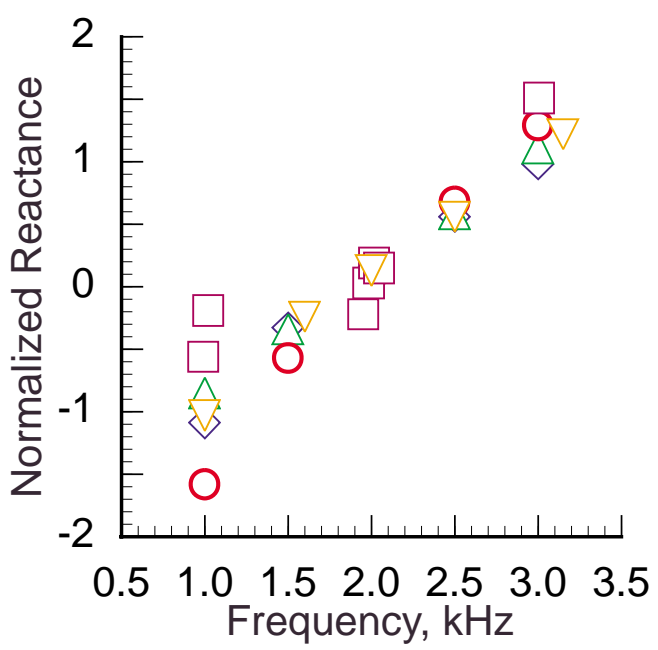

Figure 10. Liner 1 Reactance, $M=0.3$.

Broadband Sound in Ducts," Noise Control Engineering Journal, 1995.

8. Premo, J., "The Application Of A Time-Domain Model To Investigate The Impedance Of Perforate Liners Including The Effects Of Bias Flow," AIAA 99-1876, 1999.

9. Watson, W. R., Jones, M. G., and Parrott, T. L., "Validation of an Impedance Eduction Method in Flow," AIAA Journal, Vol. 37, No. 7, 1999.

10. Armstrong, D. L., Beckemeyer, R. J. and Olsen, R. F., "Impedance Measurements of Acoustic Duct Liners With Grazing Flow," Paper presented at the $87^{\text {th }}$ Meeting of the Acoustical Society of America, New York, NY, 1974.

11. Watson, W.R., Tracy, M.B., Jones, M.G. and Parrott, T.L., "Impedance Eduction in the Presence of Shear Flow," AIAA 2001-2236, 2001.

12. Jones, M.G., Watson, W.R., Tracy, M.B. and Parrott, T.L., "Comparison of Two Acoustic Waveguide Methods for Determining Liner Impedance," AIAA 2001-2266, 2001.

13. Myers, M. K., "On the Acoustic Boundary Condition in the Presence Of Flow," Journal Of Sound And Vibration, Vol. 71, No. 3, 1980, pp. 429-434.

14. Gallman, J.M. and Kunze, R.K., "Grazing Flow Acoustic Impedance Testing for the NASA AST Program," AIAA 2002-2447, 2002.

15. Bielak, G., Gallman, J., Kunze, R., Murray, P., Premo, J., Kosanchick, M., Hersh, A., Celano, J., 
Walker, B., Yu, J., Kwan, H.W., Chiou, S., Kelly, J., Betts, J., Follet, J., and Thomas, R., "Advanced Nacelle Acoustic Lining Concepts Development," NASA CR-2002-211672, 2002.

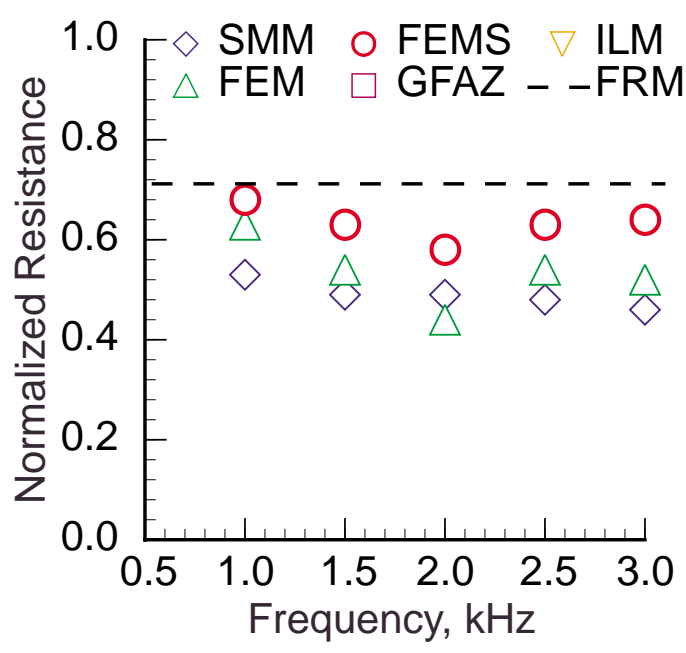

Figure 11. Liner 3 Resistance, $M=0.3$.

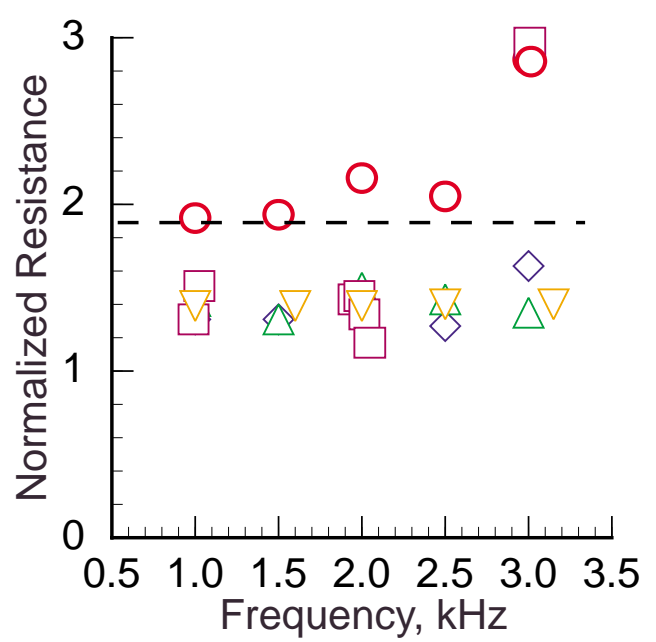

Figure 12. Liner 1 Resistance, $\mathrm{M}=0.5$.
16. Syed, A.A., Yu, J., Kwan, H.W. and Chien, E., "The Steady Flow Resistance of Perforated Sheet Materials in High Speed Grazing Flows," NASA CR-2002211749, 2002.

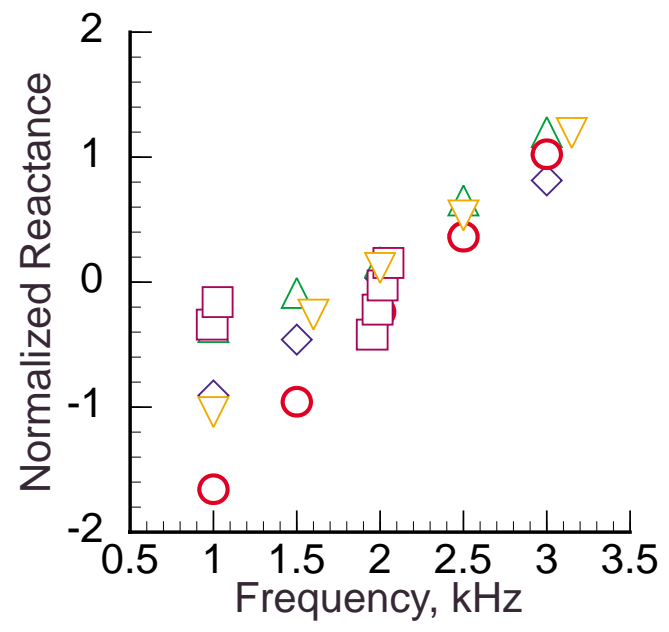

Figure 13. Liner 1 Reactance, $M=0.5$.

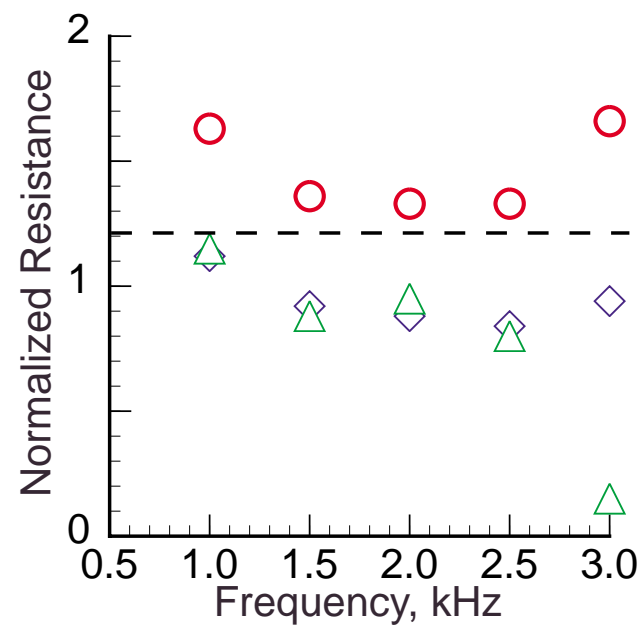

Figure 14. Liner 3 Resistance, $M=0.5$. 


2(a) M=0.3
\begin{tabular}{|c||c|c|c|c|c||c|c|c|c|c|}
\hline Freq, Hz & SMM & FEM & FEMS & GFAZ & ILM & SMM & FEM & FEMS & GFAZ & ILM \\
\hline 992 & & & & 1.49 & & & & & -0.56 & \\
\hline 1000 & 0.82 & 0.91 & 1.05 & & 0.85 & -1.09 & -0.85 & -1.58 & & -1.03 \\
\hline 1024 & & & & 1.64 & & & & & -0.19 & \\
\hline 1500 & 0.76 & 0.83 & 0.97 & & & -0.33 & -0.34 & -0.57 & & \\
\hline 1600 & & & & & 0.86 & & & & & -0.24 \\
\hline 1952 & & & & 1.45 & & & & & -0.22 & \\
\hline 1984 & & & & 1.37 & & & & & 0.03 & \\
\hline 2000 & 0.74 & 0.79 & 0.94 & & 0.86 & 0.13 & 0.18 & 0.08 & & 0.13 \\
\hline 2016 & & & & 1.20 & & & & & 0.19 & \\
\hline 2048 & & & & 1.15 & & & & & 0.15 & \\
\hline 2500 & 0.81 & 0.83 & 1.07 & & 0.86 & 0.56 & 0.58 & 0.68 & & 0.56 \\
\hline 2976 & & & & 4.32 & & & & & 4.58 & \\
\hline 3000 & 0.71 & 0.74 & 0.98 & & & 0.98 & 1.11 & 1.29 & & \\
\hline 3008 & & & & 0.63 & & & & & 1.51 & \\
\hline
\end{tabular}

2(b) M=0.5
\begin{tabular}{|c||c|c|c|c|c||c|c|c|c|c|}
\hline Freq, Hz & SMM & FEM & FEMS & GFAZ & ILM & SMM & FEM & FEMS & GFAZ & ILM \\
\hline 992 & & & & 1.31 & & & & & -0.34 & \\
\hline 1000 & 1.31 & 1.42 & 1.92 & & 1.39 & -0.91 & -0.36 & -1.66 & & -1.04 \\
\hline 1024 & & & & 1.51 & & & & & -0.16 & \\
\hline 1500 & 1.31 & 1.31 & 1.94 & & & -0.46 & -0.09 & -0.96 & & \\
\hline 1600 & & & & & 1.39 & & & & & -0.26 \\
\hline 1952 & & & & 1.43 & & & & & -0.42 & \\
\hline 1984 & & & & 1.45 & & & & & -0.22 & \\
\hline 2000 & 1.41 & 1.50 & 2.16 & & 1.39 & 0.03 & 0.16 & -0.24 & & 0.11 \\
\hline 2016 & & & & 1.34 & & & & & -0.03 & \\
\hline 2048 & & & & 1.17 & & & & & 0.15 & \\
\hline 2500 & 1.27 & 1.43 & 2.05 & & 1.40 & 0.38 & 0.65 & 0.36 & & 0.53 \\
\hline 2976 & & & & 6.62 & & & & & 7.36 & \\
\hline 3000 & 1.63 & 1.35 & 2.87 & & & 0.81 & 1.20 & 1.02 & & \\
\hline 3008 & & & & 2.97 & & & & & 3.77 & \\
\hline
\end{tabular}

Table 2. Liner 1 Normalized Impedance

(FRM $\theta=1.12$ and 1.89 at $M=0.3$ and 0.5 , respectively)

3(a) M=0.3
\begin{tabular}{|c||c|c|c||c|c|c|}
\hline Freq, Hz & SMM & FEM & FEMS & SMM & FEM & FEMS \\
\hline 1000 & 1.25 & 1.33 & 1.60 & -1.12 & -0.82 & -1.63 \\
\hline 1500 & 1.19 & 1.24 & 1.53 & -0.37 & -0.30 & -0.63 \\
\hline 2000 & 1.16 & 1.07 & 1.52 & 0.14 & 0.28 & 0.06 \\
\hline 2500 & 1.14 & 1.09 & 1.55 & 0.70 & 0.63 & 0.86 \\
\hline 3000 & 1.40 & 1.12 & 1.97 & 1.27 & 1.20 & 1.66 \\
\hline
\end{tabular}




3(b) M=0.5
\begin{tabular}{|c||c|c|c||c|c|c|}
\hline Freq, Hz & SMM & FEM & FEMS & SMM & FEM & FEMS \\
\hline 1000 & 2.22 & 2.02 & 3.26 & -0.73 & -0.14 & -1.51 \\
\hline 1500 & 2.13 & 1.92 & 3.13 & -0.58 & -0.10 & -1.27 \\
\hline 2000 & 2.15 & 2.31 & 3.34 & 0.07 & -0.03 & -0.35 \\
\hline 2500 & 2.05 & 1.92 & 3.45 & 0.65 & 0.84 & 0.59 \\
\hline 3000 & 2.31 & 1.58 & 4.21 & 1.06 & 1.01 & 1.16 \\
\hline
\end{tabular}

Table 3. Liner 2 Normalized Impedance

(FRM $\theta=1.62$ and 2.71 at $M=0.3$ and 0.5 , respectively)

4(a) M=0.3
\begin{tabular}{|c||c|c|c||c|c|c|}
\hline Freq, Hz & SMM & FEM & FEMS & SMM & FEM & FEMS \\
\hline 1000 & 0.53 & 0.63 & 0.68 & -1.14 & -0.93 & -1.64 \\
\hline 1500 & 0.49 & 0.54 & 0.63 & -0.46 & -0.42 & -0.75 \\
\hline 2000 & 0.49 & 0.44 & 0.58 & 0.00 & 0.04 & 0.06 \\
\hline 2500 & 0.48 & 0.54 & 0.63 & 0.39 & 0.45 & 0.47 \\
\hline 3000 & 0.46 & 0.52 & 0.64 & 0.80 & 1.03 & 1.06 \\
\hline
\end{tabular}

4(b) $M=0.5 \quad$ Resistance Reactance

\begin{tabular}{|c||c|c|c||c|c|c|}
\hline Freq, $\mathrm{Hz}$ & SMM & FEM & FEMS & SMM & FEM & FEMS \\
\hline 1000 & 1.12 & 1.15 & 1.63 & -1.05 & -0.41 & -1.86 \\
\hline 1500 & 0.92 & 0.88 & 1.36 & -0.45 & -0.23 & -0.94 \\
\hline 2000 & 0.88 & 0.95 & 1.33 & -0.02 & 0.06 & -0.25 \\
\hline 2500 & 0.84 & 0.80 & 1.33 & 0.32 & 0.51 & 0.36 \\
\hline 3000 & 0.94 & 0.15 & 1.66 & 0.80 & 0.93 & 1.21 \\
\hline
\end{tabular}

Table 4. Liner 3 Normalized Impedance (FRM $\theta=0.71$ and 1.22 at $M=0.3$ and 0.5 , respectively)

5(a) $\mathrm{M=0.3}$ Resistance Reactance

\begin{tabular}{|c||c|c|c||c|c|c|}
\hline Freq, Hz & SMM & FEM & FEMS & SMM & FEM & FEMS \\
\hline 1000 & 0.68 & 0.87 & 0.88 & -1.15 & -0.89 & -1.66 \\
\hline 1500 & 0.61 & 0.65 & 0.77 & -0.43 & -0.39 & -0.71 \\
\hline 2000 & 0.56 & 0.47 & 0.69 & 0.11 & 0.13 & 0.07 \\
\hline 2500 & 0.48 & 0.51 & 0.64 & 0.54 & 0.53 & 0.67 \\
\hline 3000 & 0.53 & 0.48 & 0.74 & 1.07 & 0.98 & 1.43 \\
\hline
\end{tabular}

5(b) $\mathrm{M}=\mathbf{0 . 5} \quad$ Resistance Reactance

\begin{tabular}{|c||c|c|c||c|c|c|}
\hline Freq, Hz & SMM & FEM & FEMS & SMM & FEM & FEMS \\
\hline 1000 & 1.41 & 1.52 & 2.05 & -1.10 & -0.29 & -1.96 \\
\hline 1500 & 1.25 & 1.15 & 1.85 & -0.41 & -0.12 & -0.90 \\
\hline 2000 & 1.06 & 1.17 & 1.63 & 0.09 & 0.09 & -0.09 \\
\hline 2500 & 1.02 & 1.08 & 1.67 & 0.45 & 0.66 & 0.53 \\
\hline 3000 & 1.03 & 1.69 & 1.87 & 0.95 & 1.91 & 1.45 \\
\hline
\end{tabular}

Table 5. Liner 4 Normalized Impedance

(FRM $\theta=0.83$ and 1.46 at $M=0.3$ and 0.5 , respectively) 